UDK 528.482

\title{
USING SMART PHONES FOR DEFORMATIONS MEASUREMENTS OF STRUCTURES
}

\author{
Khalid L. A. EL-ASHMAWY ${ }^{1,2}$ \\ ${ }^{1}$ Department of Civil Engineering, Al-Matria Faculty of Engineering, Helwan University, \\ Matria, 11718 Cairo, Egypt \\ ${ }^{2}$ Department of Civil Engineering, College of Engineering and Islamic Architecture, \\ Umm Al-Qura University, Makkah, Saudi Arabia \\ E-mail:khalid85_2002@yahoo.com
}

Received 11 January 2017; accepted 10 May 2017

\begin{abstract}
The present work tests the suitability of using the digital cameras of smart phones for close range photogrammetry applications. For this purpose two cameras of smart phones Lumia 535 and Lumia 950 XL were used. The resolutions of the two cameras are 5 and 20 Mpixels respectively. The tests consist of (a) self calibration of the two cameras, (b) the implementation of close-range photogrammetry using the cameras of the two smart phones, theodolite intersection with LST method, and linear variable displacement transducers (LVDTs) for the measurement of vertical deflections, and (c) accuracy of photogrammetric determination of object space coordinates. The results of using Lumia $950 \mathrm{XL}$ are much better than using Lumia 535 and are better or comparable to the results of theodolite intersection with least squares technique (LST). Finally, it can be stated that the digital cameras of smart phones are suitable for close range photogrammetry applications according to accuracy, costs and flexibility.
\end{abstract}

Keywords: smart phones, close range photogrammetry, self calibration block adjustment, deflection, intersection with least squares technique (LST).

\section{Introduction}

Photogrammetry is used to determine three-dimensional coordinates of an object from two-dimensional photographs. Traditional photogrammetry is based on the use of precisely cameras known as metric cameras which have the following properties:

- They are designed for photogrammetric applications only and take large format photographic images on film or glass plates.

- Their interior orientations are stable. They have rigid lens cone and the focal distance is set at the factory. The fiducial marks are fixed to the camera and are printed on each exposure as reference points.

- They have low distortion lens and can be obtained and characterized through the camera calibration procedures.

- The film is flat by incorporating a film flattening device such as a pressure plate or vacuum system.
The demerits of metric cameras are high prices, the limitation of the focus range and unsuitability for all photogrammetric applications due to the limitation in their design. For these demerits, the photogrammetrists prefer non-metric cameras.

Generally, non-metric cameras do not attain any of the desired properties listed above for metric camera. However, their main advantages are readily available, low prices, light in weight, and capable of interchanging lenses and focusing for camera-object distance. The well-known disadvantages of non-metric cameras are:

-Instability of interior orientation where the focal length may be changed for each exposure and the direction of the optical axis may alter with focusing movements.

- Unavailability of the fiducial marks.

- Irregularity of radial and tangential lens distortions. These are more difficult to compensate and formulate during the computations of object space positions. 
- The lack of film flatness and may vary from frame to frame causing difficulties in its correcting during the computation of the object space coordinates.

With the recent revolution in manufacturing digital cameras, the photogrammetrists turned their attention to the use of digital cameras in photogrammetric applications.

In the digital cameras, digital sensors removes the need for fiducial marks because the array of pixels can be used to define the image coordinate system. In spite of any digital camera contains a measuring system of image coordinates, it is agreed that the term "metric camera" should be reserved for those cameras specifically designed for photogrammetric tasks, such as the Rollei D7 Metric (Khalaf, Yassin 2016).

Digital cameras can be classified into two categories according to their metric potential: professional or high quality grade cameras and consumer or low cost grade cameras. Professional cameras have properties such as good lens quality, a robust structure, a large sensor with high resolution and sensitivity, the ability to exchange lenses, while consumer, or amateur, grade cameras include models that may include any of these features to compact cameras where the lens is packaged together inside the camera.

The main important difference between consumer cameras and professional cameras is the lower geometric stability of amateur cameras. This issue involves lower reliability and durability over time of the modelization of the internal geometry of the cameras. To solve these limitations mathematical models and rapid calibration procedures have been developed (Khalaf, Yassin 2016) in recent years that allow these cameras to be used for photogrammetric applications (Udin et al. 2014; Sanz-Ablanedo et al. 2009; Abd Elbasit et al. 2009).

Recently, smartphones are widely used and are available to everyone. Smartphone is a mobile device having mobile phone functions such as personal information management, compass, digital camera, a GNSS receiver, accelerometers, and Internet communication. Comparing between the different hardware and software components, smartphones are more suitable components for acquisition of image data because they are very cheap, portable and can take images of up to 20 mega pixels resolution. Researches on using built-in cameras of smartphones for photogrammetric applications are in progress (Zhang et al. 2016; Teoa et al. 2014; Bakuła, Flasiński 2013).
This study aims to use the digital camera of the smartphone for the determination of the deformation of structures applying the principles of close range photogrammetry. To evaluate the accuracy of using the smart phones, the obtained results will be compared with the results of theodolite intersection with least squares technique (LST) method (El-Ashmawy 2017).

\section{Data acquisition and processing}

An I-shaped steel beam was used to determine its deflection response under static load. The beam of dimensions $2.0 \mathrm{~m}$ length, $100 \mathrm{~mm}$ depth, $100 \mathrm{~mm}$ flange width, $6 \mathrm{~mm}$ web thickness, and $8 \mathrm{~mm}$ flange thickness was placed on hydraulic jack system.

The beam was vertically supported close to its two ends while the loading jack was located at the centre of the beam. Three values of the load $0.5,1.0$ and 1.5 ton were applied. Measurements with LVDTs, total station and close range photogrammetry were taken at each load stage. Furthermore, measurements before loading at the beginning of the experiment were captured to allow comparison with the subsequent measurements.

Five deflection points were chosen at distances of $\mathrm{L} / 5,2 \mathrm{~L} / 5, \mathrm{~L} / 2,3 \mathrm{~L} / 5$ and $4 \mathrm{~L} / 5$ on the beam. Five paper prisms were put on the deflection points as shown in Figure 1.

A total number of 20 paper prisms were selected on the stable components of the test frame and concrete blocks around the beam to constitute a stable reference of control points for photogrammetric processing.

Two observation stations (control points) were chosen on the floor $5 \mathrm{~m}$ apart from the beam. The difference in levels between the two stations was

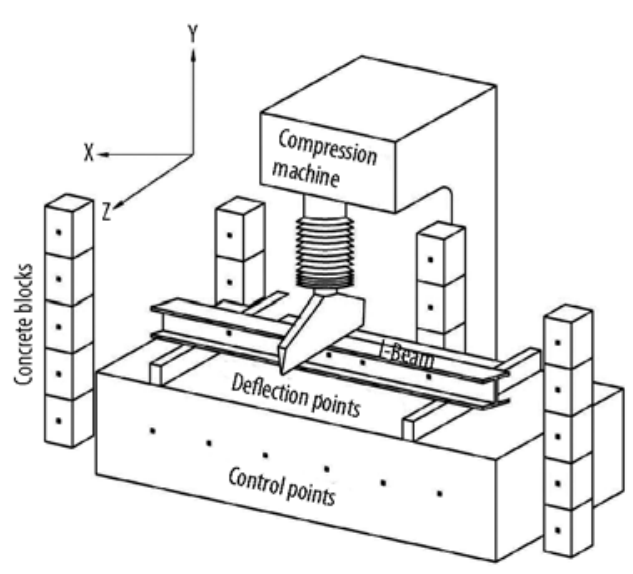

Fig. 1. The beam, loading, photogrammetric control points and locations of the deflection points 
determined with the precise leveling using Leica NA2 automatic level with a Leica $(10 \mathrm{~mm})$ GPM3 parallel plate micrometer attachment and a GPLE3 geodetic invar staff with $10 \mathrm{~mm}$ graduations. The horizontal distance between the two stations was measured using Topcon GTS710 total station. The horizontal distance was measured six times, 3 direct and 3 reverse, and was taken as the mean of the six measured values. According to the measured horizontal distance and the height difference between the two observation stations, the coordinates of the two stations were determined.

The beam deflections were determined by three different methods as explained below.

The first method consisted of direct measurement of the deflections, before loading the beam and after each loading stage, using LVDTs.

The second method included theodolite intersection using LST method (El-Ashmawy 2017). The observations of the deflection points were taken before loading the I-beam and after each stage of loading, and observations of photogrammetric control points were taken once before loading the beam. The points were observed from the two observation stations using Wild (Lieca) T2 with 1" least count. The horizontal angles to the points were observed by direction and closing the horizon methods and reading the horizontal circle in both the left and right faces. Multiple observations of the angle are made, with the circle being advanced prior to each reading to compensate for the systematic errors. Each angle was determined for each observations set and the final horizontal angle value was taken as the average of all measured values. The vertical angle of each point was determined from the vertical circle readings in both left and right faces and taking the mean value. All observations were entered to a developed software utilizing LST (El-Ashmawy 2017) to compute the adjusted coordinates of points and to export the results to an ASCII file to be processed and analyzed.

The third method involved the use of close range photogrammetry. Two smartphones, Lumia 535 and Lumia 950-XL with digital cameras, were employed. The specifications for the digital cameras of the two smartphones are shown in Table 1.

Accurate camera configuration and orientation are necessary prerequisites for achieving high geometry of the intersected angles and for the extraction of precise and reliable 3D metric information from images. High geometry of intersected angles can be obtained when the base distance to the object distance $(\mathrm{B} / \mathrm{H})$ ratio is ranging from 0.5 to 0.9 (Hasegawa et al. 2000).
Photos were taken at a distance camera-object of $2.0 \mathrm{~m}$. For each load stage (zero load, 0.5, 1.0 and 1.5 ton) 3 photos were taken: 1 vertical photo and 2 oblique photos taken from the edges of the beam. Prior and after the experiment photos were taken for use in the camera calibration process.

Table 1. Specifications of the used digital cameras

\begin{tabular}{|l|c|c|}
\hline Sensor & Lumia 535 & Lumia 950-XL \\
\hline Pixel size & $\begin{array}{c}1 / 4^{\prime \prime} \\
3.6 \times 2.7 \mathrm{~mm}\end{array}$ & $\begin{array}{c}1 / 2.4^{\prime \prime} \\
5.92 \times 4.44 \mathrm{~mm}\end{array}$ \\
\hline Image format & $\begin{array}{c}2560 \times 1920 \\
5 \text { megapixels }\end{array}$ & $\begin{array}{c}4992 \times 3744 \\
20 \text { megapixels }\end{array}$ \\
\hline Lens & Carl Zeiss optics & Carl Zeiss optics \\
\hline Focal length & $\begin{array}{c}28 \mathrm{~mm} \\
(35 \mathrm{~mm} \text { equivalent })\end{array}$ & $\begin{array}{c}26 \mathrm{~mm} \\
(35 \mathrm{~mm} \text { equivalent })\end{array}$ \\
\hline Auto focus & Yes & Yes \\
\hline Aperture & F2.4 & F1.9 \\
\hline Output format & Only JPEG & JPEG, DNG \\
\hline $\begin{array}{l}\text { Camera minimum } \\
\text { focus range }\end{array}$ & $10 \mathrm{~cm}$ & $10 \mathrm{~cm}$ \\
\hline
\end{tabular}

Photogrammetric measurements are performed on the taken images. The generated images by cameras are downloaded and processed as digital files by the computer. The measurement of coordinates of digital files are mainly performed on a computer display. Presently, the measurement technologies allow precise determination of image coordinates.

The coordinate measurement of image points was carried out manually using the options of AutoCAD software (Autodesk 2012). The image was displayed in raster format and scaled to its size in pixels. In this case, AutoCAD can measure the image coordinates of points to a least count of 0.0001 pixel. For automatic reading and storing the coordinates of image points, a program written in AutoLisp (version of Common Lisp language with some additional features unique to AutoCAD) (Autodesk 2013) developed by the author was used.

Processing the photogrammetric and control data collected during the experiment was performed using Col_Cal_Consts software (El-Ashmawy, Azmi 2003). This software utilizes collinearity equations and performs self-calibration block adjustment. The software is suitable for processing aerial and close-range photogrammetric blocks and provides an access to major computational phases of analytical block triangulation. The main functions of the used software are: 
a. Data preparation: It performs the necessary tasks for preparing the data to start block adjustment such as:

- Testing the geometry of the input data: It includes testing the availability of each point in at least two photographs and giving complete information about the block adjustment parameters such as number of observations and unknowns, degree of freedom, etc.

- Two dimensional affine transformation to transform the instrumental photo coordinates of control, pass and tie points to the fiducial marks coordinate system.

- Refinement of photo coordinates: Corrections for principal point displacement, lens distortion, atmospheric refraction and earth curvature, if applicable, are to be applied to the photo coordinates.

- Computation of the initial values of unknowns (El-Ashmawy 1999): It includes the computation of the initial values of camera exterior orientation parameters and/or object space coordinates of points which are essential for starting the iterative solution.

b. Iterative least squares method solution for simultaneous block adjustment. This includes the computations of the adjusted values of camera interior and exterior orientations parameters, object space coordinates of control and pass points, residuals of photo and object space coordinates of control points, and variance of unit weight.

c. Computation of statistical data: It includes the computation of the necessary data for statistical analysis and error detection (El-Ashmawy 1999) such as variance of unit weight, cofactor and covariance matrices for unknowns, adjusted photo coordinates and their cofactor matrix, residuals of photo coordinates, dimensions of error ellipses, etc.

Col_Cal_Consts software computes the corrected photo coordinates $\left(x_{p} \& y_{p}\right)$ using the following equations (Ghosh 1988):

$$
\left.\begin{array}{l}
x_{p}=\bar{x}_{p}-x_{o}+F(k) \bar{x}_{p} / r+\Delta x(p) \\
y_{p}=\bar{y}_{p}-y_{o}+F(k) \bar{y}_{p} / r+\Delta y(p)
\end{array}\right\},
$$

in which

$x_{p}, y_{p}$ are the corrected photo coordinates;

$\bar{x}_{p}, \bar{y}_{p}$ are the observed photo coordinates;

$x_{o}, y_{o}$ are the photo coordinates of the principal point;

$$
\begin{gathered}
r^{2}=\bar{x}_{p}^{2}+\bar{y}_{p}^{2} \\
F(k)=K_{1} r^{3}+K_{2} r^{5}+K_{3} r^{7}=
\end{gathered}
$$

function of symmetrical radial lens distortion;

$\Delta x(p), \Delta y(p)$ are the decentering lens distortion corrections for $x$ and $y$ photo coordinates, and can be obtained by:

$$
\left.\begin{array}{l}
\Delta x(p)=P_{1}\left(r^{2}+2 \bar{x}_{p}^{2}\right)+P_{2}\left(2 \bar{x}_{p} \bar{y}_{p}\right) \\
\Delta y(p)=P_{2}\left(r^{2}+2 \bar{y}_{p}^{2}\right)+P_{1}\left(2 \bar{x}_{p} \bar{y}_{p}\right)
\end{array}\right\},
$$

where $P_{1}, P_{2}$ are correction coefficients for decentering lens distortion.

From the above equations, the parameters of interior orientation that are solved for by Col_Cal_Consts software include:

- Camera Focal Length;

- Coordinates of the Principal Point $\left(x_{o}, y_{o}\right)$;

- Coefficients of radial distortions $\left(K_{1}, K_{2}, K_{3}\right)$;

- Coefficients of decentring distortions $\left(P_{1}, P_{2}\right)$.

Photogrammetric and control data are entered to the Col_Cal_Consts software by editing a suitable data file and the software generates and stores its results in special files (in ASCII format) for later processing.

\section{Results}

\section{a. Calibration of the digital cameras}

Self calibration processing of the photogrammetric and geodetic data was performed separately for each loading stage and using each camera and the results are shown in Table 2. Each least squares adjustment contained 210 observation equations (coordinates of 20 control points and coordinates of 75 image points) and 101 unknowns (coordinates of 20 control and 5 new points, 8 elements of interior orientation and 18 elements of exterior orientation). Accordingly, the degree of freedom was 109. The root mean square error (RMSE) of the measured image coordinates was about $\pm 1 \mu \mathrm{m}$ (0.8 pixel) for lumia $950 \mathrm{XL}$ which is much better than using lumia 535 ( $\pm 2 \mu \mathrm{m} \sim 1.4$ pixel).

Table 2 shows clearly that the obtained accuracies in object space coordinates of control points are within sub-millimeter.

Lumia 950 XL has a sensor of 20 Mega pixels. It is 4 times larger than the sensor of Lumia 535. This leads to theoretical expectations that Lumia $950 \mathrm{XL}$ may give an accuracy of factor 2.0 ( better compared to Lumia 535. Lumia $950 \mathrm{XL}$ almost strictly meets this expectation by giving 2.1 to 2.3 times better numbers than the Lumia 535. 
The most important step before using the smartphone camera for photogrammetric applications is to test the stability of its camera. The cameras had to pass a stability analysis procedure, which served to verify that the estimated interior orientation parameters do not change significantly over time. The stability analysis procedure is necessary because the cameras are not originally designed for metric applications.

The stability test was performed by two methods. The first method consisted of using only the twenty control points and Col_Cal_Consts software for getting and comparing the camera interior orientation parameters before and after the experiment. The comparison between the obtained values of the camera interior orientations showed the stability of the camera characteristics in time.
The second method involved the analyses of the shown results in Table 2 where there are no significant changes in the standard deviation values for measuring the image coordinates or determination of object space coordinates of control points in the different observation stages for each smart phone. This definitely indicates the stability of interior orientation of both cameras of the two smart phones.

\section{b. Deflection determinations}

Subtracting the $\mathrm{Y}$ coordinate of each deflection point before loading the beam from the $\mathrm{Y}$ coordinate of the same deflection point, as shown in Figure 1, for each loading stage determines the beam deflections $(\delta \mathrm{Y})$ at each point for each loading stage.

Table 2. The results of simultaneous self calibration block adjustment

\begin{tabular}{|c|c|c|c|c|c|c|c|c|c|c|c|}
\hline \multirow[b]{2}{*}{ 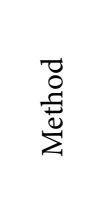 } & \multirow{2}{*}{$\begin{array}{l}n \\
0 \\
0 \\
\frac{1}{2} \\
2 \\
0 \\
0 \\
\dot{z}\end{array}$} & \multirow{2}{*}{ 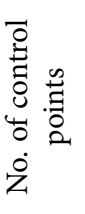 } & \multirow{2}{*}{ 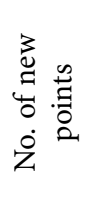 } & \multirow{2}{*}{ 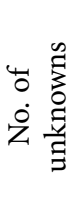 } & \multirow{2}{*}{ 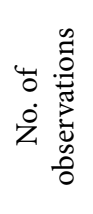 } & \multirow{2}{*}{ 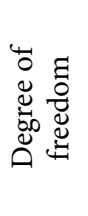 } & \multirow{2}{*}{ 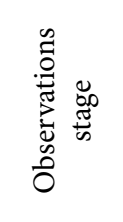 } & \multirow{2}{*}{ 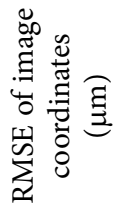 } & \multicolumn{3}{|c|}{ Standard deviation $(\mathrm{mm})$} \\
\hline & & & & & & & & & $\mathrm{X}$ & |Y & Z \\
\hline \multirow{4}{*}{$\begin{array}{l}\text { Lumia } \\
535\end{array}$} & \multirow{4}{*}{3} & \multirow{4}{*}{20} & \multirow{4}{*}{5} & \multirow{4}{*}{101} & \multirow{4}{*}{210} & \multirow{4}{*}{109} & $\begin{array}{c}\text { Before } \\
\text { Loading }\end{array}$ & 2.04 & 0.180 & 0.183 & 0.268 \\
\hline & & & & & & & Stage 1 & 1.99 & 0.179 & 0.180 & 0.263 \\
\hline & & & & & & & Stage 2 & 2.05 & 0.188 & 0.185 & 0.271 \\
\hline & & & & & & & Stage 3 & 2.08 & 0.185 & 0.182 & 0.262 \\
\hline \multirow{4}{*}{$\begin{array}{l}\text { Lumia } \\
950 \mathrm{XL}\end{array}$} & \multirow{4}{*}{3} & \multirow{4}{*}{20} & \multirow{4}{*}{5} & \multirow{4}{*}{101} & \multirow{4}{*}{210} & \multirow{4}{*}{109} & $\begin{array}{l}\text { Before } \\
\text { Loading }\end{array}$ & 0.96 & 0.085 & 0.090 & 0.124 \\
\hline & & & & & & & Stage 1 & 1.03 & 0.091 & 0.095 & 0.128 \\
\hline & & & & & & & Stage 2 & 1.05 & 0.092 & 0.094 & 0.121 \\
\hline & & & & & & & Stage 3 & 0.97 & 0.089 & 0.092 & 0.126 \\
\hline
\end{tabular}

Table 3. Values of vertical deflections and RMSEs (in mm)

\begin{tabular}{|c|c|c|c|c|c|c|c|c|c|c|c|c|}
\hline $\begin{array}{l}\text { Loading } \\
\text { Stage }\end{array}$ & \multicolumn{4}{|c|}{$\begin{array}{l}\text { Stage } 1 \\
\text { 1.0 Ton }\end{array}$} & \multicolumn{4}{|c|}{$\begin{array}{l}\text { Stage } 2 \\
1.5 \text { Ton }\end{array}$} & \multicolumn{4}{|c|}{$\begin{array}{l}\text { Stage } 3 \\
2.0 \text { Ton }\end{array}$} \\
\hline$\dot{\leftrightarrow} \ddot{\Xi}$ & 莺 & 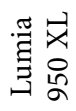 & 总 & $\stackrel{\infty}{\stackrel{\infty}{a}}$ & 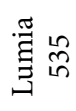 & 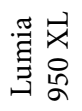 & 离. 晜 & $\stackrel{\infty}{\stackrel{\infty}{a}}$ & 莺 & 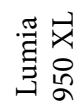 & 离. & $\stackrel{n}{\stackrel{n}{a}}$ \\
\hline $\mathrm{L} / 5$ & 1.67 & 1.42 & 1.59 & 1.21 & 3.79 & 3.52 & 3.52 & 3.25 & 5.82 & 5.66 & 5.42 & 5.13 \\
\hline $2 \mathrm{~L} / 5$ & 2.96 & 2.82 & 2.75 & 2.58 & 5.35 & 5.19 & 5.32 & 4.91 & 8.89 & 8.74 & 8.95 & 8.35 \\
\hline $\mathrm{L} / 2$ & 4.17 & 4.01 & 4.09 & 3.72 & 8.06 & 7.72 & 7.94 & 7.43 & 11.83 & 11.52 & 11.98 & 11.24 \\
\hline $3 \mathrm{~L} / 5$ & 2.64 & 2.37 & 2.65 & 2.28 & 5.74 & 5.42 & 5.71 & 5.13 & 9.08 & 9.01 & 8.72 & 8.57 \\
\hline $4 \mathrm{~L} / 5$ & 1.56 & 1.42 & 1.62 & 1.13 & 3.46 & 3.34 & 3.56 & 3.11 & 6.14 & 5.75 & 6.21 & 5.36 \\
\hline RMSE & 0.42 & 0.24 & 0.37 & - & 0.52 & 0.27 & 0.46 & - & 0.63 & 0.31 & 0.59 & - \\
\hline $\begin{array}{l}\text { RMSE for } \\
\text { all observa- } \\
\text { tions }\end{array}$ & 0.53 & 0.27 & 0.48 & - & & & & & & & & \\
\hline
\end{tabular}


The obtained $\delta \mathrm{Y}$ and the readings of the LVDTs of the deflection points for each loading stage using the different observing methods are shown in Table 3.

Values of RMSE are computed based on the discrepancies. Considering the values of measured deflections using LVDTs are errorless, the discrepancies of deformations were computed by subtracting the obtained deformations using LVDTs from the deformations obtained by the Lumia 535, Lumia 960 XL or intersection method.

The maximum deflection occurred at mid-span directly below the point of the force load and the deflection decreased near the ends where the beam was supported. The maximum deflection values using LVDTs were $3.72 \mathrm{~mm}, 7.43 \mathrm{~mm}$ and $11.24 \mathrm{~mm}$ for increments of 1.0 ton, 1.5 ton and 2.0 ton respectively.

Comparing the values of RMSE, the following conclusion can be drawn:

- The results of close range photogrammetry using Lumia 535 and intersection method are of comparable accuracy.

- The more accurate results can be obtained by close range photogrammetry using Lumia 950 XL.

- The camera resolution has a significant effect on improving the accuracy of the obtained deflection.

\section{c. Object space coordinates accuracy}

Furthermore, values of RMSE for the difference in the results of object space coordinates of deflection points determination and the results of the intersection method were calculated and shown in Table 4 .

Table 4. Values of RMSEs for object space coordinates of deflection points (in $\mathrm{mm}$ )

\begin{tabular}{|c|c|c|c|}
\hline Method & X & Y & Z \\
\hline Lumia 535 & 0.42 & 0.39 & 0.54 \\
\hline Lumia 960 XL & 0.27 & 0.25 & 0.32 \\
\hline
\end{tabular}

From Table 4, it is very clear that the accuracy of Lumia $950 \mathrm{XL}$ is much better than that of Lumia 535.

\section{Conclusions}

This paper presented the use of digital cameras of smart phones for close range photogrammetry applications. The metric accuracies of two consumergrade digital cameras of two smart phones (Lumia 535 and Lumia $950 \mathrm{XL}$ ) were tested and presented in this paper.
The test consisted of (a) self calibration of the two cameras, (b) the implementation of close-range photogrammetry using the cameras of the two smart phones, theodolite intersection with LST method, and LVDTs for the measurement of vertical deflections at specified points in steel beam under a concentrated load of 1.0, 1.5 or 2.0 ton in its mid span, and (c) accuracy of photogrammetric determination of object space coordinates.

Self calibration processing of the photogrammetric and geodetic data showed that RMSE values of the measured image coordinates was about $\pm 1 \mu \mathrm{m}(0.8$ pixel) for Lumia $950 \mathrm{XL}$ which is much better than using Lumia 535 ( $\pm 2 \mu \mathrm{m} \sim 1.4$ pixel) and the obtained accuracies in object space coordinates of control points are within sub-millimeter. Furthermore, there are no significant changes in the standard deviation values for measuring the image coordinates or determination of object space coordinates of control points in the different observation stages for each smart phone. This definitely indicates the stability of interior orientation of both cameras of the two smart phones. Finally, the results of using Lumia $950 \mathrm{XL}$ are much better than using Lumia 535.

The deflection determination test showed that the results of close range photogrammetry using Lumia 535 and intersection with LST method are of comparable accuracy and more accurate results can be obtained using Lumia 950 XL. Accordingly, the camera resolution has a significant effect on improving the accuracy of the obtained deflection. In spite of close range photogrammetry using Lumia 535 and intersection with LST method give similar results, the acquisition of measurements from theodolite(s) is very slow and limited number of deflection points can only be measured.

The results of photogrammetric determination of object space coordinates of points showed that the accuracy of Lumia $950 \mathrm{XL}$ is much better than that of Lumia 535.

Finally, it can be stated that the digital cameras of smart phones are suitable for close range photogrammetry applications according to accuracy, costs and flexibility.

\section{References}

Abd Elbasit, M. A. M.; Saito, T.; Yasuda, H.; Anyoji, H. 2009. Using of close range photogrammetry for interrill soil erosion quantification, Journal of Arid Land Studies 19(1): 283-286.

Autodesk. 2013. AutoCAD 2014: AutoLISP developer's guide. Autodesk, Inc., USA. 
Autodesk. 2012. AutoCAD 2013: user's guide. Autodesk, Inc., USA.

Bakuła, K.; Flasiński, A. 2013. Capabilities of a smartphone for georeferenced 3d model creation: an evaluation, in 13th International Multidisciplinary Scientific GeoConference SGEM 2013, 16-22 June 2013, Albena, Bulgaria.

El-Ashmawy, K. L. A. 2017. Developing and testing a method for deformations Measurements of structures, Geodesy and Cartography 43(1): 35-40.

https://doi.org/10.3846/20296991.2017.1305545

El-Ashmawy, K. L. A.; Azmi, M. 2003. Photogrammetric simultaneous and self calibration block adjustments using coplanarity condition, Engineering Research Journal 87: C-85-C-101. Faculty of Engineering, University of Helwan, Egypt.

El-Ashmawy, K. L. A. 1999. A cost-effective photogrammetric system for engineering applications: PhD Thesis. Department of Civil Engineering, University of Roorkee, Roorkee, India.

Ghosh, S. K. 1988. Analytical photogrammetry. New York, USA: Pergamon Press.

Hasegawa, H.; Matsuo, K.; Koarai, M.; Watanabe, N.; Masaharu, H.; Fukushima, Y. 2000. DEM accuracy and the base to height $(\mathrm{B} / \mathrm{H})$ ratio of stereo images, International Archives of Photogrammetry and Remote Sensing, Vol. XXXIII, Part B4. Amsterdam.

Khalaf, A. Z.; Yassin, D. T. 2016. Evaluation the calibration of non-metric (digital camera) with photo modeler software, Imperial Journal of Interdisciplinary Research 2(10): 17651772.
Sanz-Ablanedo, E.; Rodríguez-Pérez, J. R.; Arias-Sánchez, P.; Armesto, J. 2009. Metric potential of a 3D measurement system based on digital compact cameras, Sensors 9: 41784194. https://doi.org/10.3390/s90604178

Teoa, T.; Wub, H.; Shihc, T.; Tsai, F. 2014. The analysis of smartphone apps in geomatics education, in The International Archives of the Photogrammetry, Remote Sensing and Spatial Information Sciences, Vol. XL-6, ISPRS Technical Commission VI Symposium, 19-21 May 2014, Wuhan, China.

Udin, W. S.; Ahmad, A.; Ismail, Z. 2014. Assessment of digital camera in mapping meandering flume using close range photogrammetric technique, Pertanika Journal of Science \& Technology 22(1): 123-138.

Zhang, S.; Shan, J.; Zhang, Z.; Yan, J.; Hou, Y. 2016. Integrating smartphone images and airborne Lidar data for complete urban building modelling, The International Archives of the Photogrammetry, Remote Sensing and Spatial Information Sciences, Volume XLI-B5.

Khalid L. A. EL-ASHMAWY (Dr) is associate professor of Surveying \& Digital Mapping at the Department of Civil Engineering, Al-Matria Faculty of Engineering, Helwan University, Egypt. His area of expertise includes photogrammetry, land surveying, space photography, digital mapping, GIS and development of surveying softwares.

Dr Khalid El-Ashmawy developed many surveying softwares which were published in national and international journals and conferences, such as SoftPhotoMap, PHOTOMAP, MATHP, ScanCal, LandSurMap, Calendar, RSA (Road Safety Assessment), and MathDTM softwares. 